\title{
Combinatorics and geometry of circular gene networks models
}

\author{
V.P. Golubyatnikov ${ }^{1,2} \bowtie$, L.S. Minushkina ${ }^{3}$
}

Abstract: We study combinatorial, topological, and geometric properties of phase portraits of nonlinear dynamical systems which simulate functioning of some simple circular gene networks. The principal aim of these studies in general is description of behavior of their trajectories in terms of qualitative theory of the ordinary differential equations in order to predict results of numerical experiments with gene networks models. Our main results concern detection and localization of periodic trajectories (or cycles), invariant domains, and invariant surfaces in these phase portraits by means of combinatorial decomposition of these portraits to elementary blocks and construction of corresponding oriented graphs, so called State Transition Diagrams. In some cases considered here and previously, these trajectories and surfaces are not stable, but they play an important role in geometry and topology of the phase portraits, as unstable equilibrium points do. In this paper, using general ideas of proof of the classical Perron-Frobenius theorem on characteristic polynomials of positive matrices, we find sufficient conditions of existence of an invariant two-dimensional surface in six-dimensional phase portrait of one dynamical system of this type. Actually, some of these conditions are necessary as well. Most of these geometric constructions and qualitative results arise from our previous joint publications with Vitaly A. Likhoshvai, see the reference list below.

Key words: dynamical systems; phase portraits; gene networks models; periodic trajectories; invariant surfaces; State Transitions Diagrams.

Acknowledgements: The work was supported by RFBR, grant No.18-01-00057. The authors express sincere gratitude for useful discussions to all our collaborators in the Project 11 of the First Workshop at the Mathematical Center in Akademgorodok, Novosibirsk, July - August 2020.

For citation: Golubyatnikov V.P., Minushkina L.S. Combinatorics and geometry of circular gene networks models. Pisma v Vavilovskii Zhurnal Genetiki i Selektsii = Letters to Vavilov Journal of Genetics and Breeding. 2020;6(4):188-192. DOI 10.18699/Letters2020-6-24

\section{Комбинаторная и геометрическая структуры моделей кольцевых генных сетей}

\author{
В.П. Голубятников ${ }^{1,2} \bowtie$, Л.С. Минушкина ${ }^{3}$
}

Аннотация: Мы изучаем комбинаторные, топологические и геометрические свойства фазовых портретов нелинейных динамических систем, которые моделируют функционирование некоторых простейших кольцевых генных сетей. Основная задача этих исследований состоит в описании поведения траекторий таких систем в терминах качественной теории обыкновенных дифференциальных уравнений с целью предсказания результатов численных экспериментов с этими моделями генных сетей. Основные результаты работы состоят в отыскании и локализации периодических траекторий (или циклов), инвариантных областей и инвариантных поверхностей в рассматриваемых фазовых портретах. Один из основных методов наших исследований основан на разбиении фазовых портретов на элементарные блоки и конструировании ориентированных графов специального вида - Диаграмм Перехода. В некоторых случаях, рассматриваемых здесь и в предыдущих публикациях, эти траектории и поверхности неустойчивы, но они играют важную роль в описании геометрии и топологии фазовых портретов указанных динамических систем, такую же, как и неустойчивые положения равновесия. В настоящей работе, используя классическую теорему Фробениуса - Перрона о характеристических многочленах положительных матриц, мы находим достаточные условия существования инвариантной двумерной поверхности в шестимерном фазовом портрете одной динамической системы рас-

\footnotetext{
${ }^{1}$ Sobolev Institute of Mathematics of the Siberian Branch of the Russian Academy of Sciences, Novosibirsk, Russia

${ }^{2}$ Yakovlev Military Institute, Novosibirsk, Russia

${ }^{3}$ Novosibirsk State University, Novosibirsk, Russia

${ }^{1}$ Институт математики им. С.Л. Соболева Сибирского отделения Российской академии наук, Новосибирск, Россия

${ }^{2}$ Новосибирский военный институт войск национальной гвардии им. И.К. Яковлева, Новосибирск, Россия

${ }^{3}$ Новосибирский национальный исследовательский государственный университет, Новосибирск, Россия

(凶) Vladimir.Golubyatnikov1@fulbrightmail.org

(C) Golubyatnikov V.P., Minushkina L.S., 2020
} 
сматриваемого типа. Некоторые из этих условий являются также и необходимыми. Основы большей части геометрических конструкций и результатов о качественном поведении траекторий таких динамических систем были заложены в наших совместных публикациях с Виталием Александровичем Лихошваем, см. список цитируемой литературы.

Ключевые слова: динамические системы; фазовые портреты; модели генных сетей; периодические траектории; инвариантные портреты; диаграммы переходов.

\section{Introduction}

Modeling of gene networks functioning by means of nonlinear dynamical systems arising from the Mass Action Law and studies of qualitative properties of solutions of these systems are investigated in numerous publications during last 60 years (Hastings et al., 1977; Banks, Mahaffy, 1978; Glass, Pasternack, 1978; Baer et al., 2006). There were also many important mathematical results related to these topics (Abraham, Robbin, 1967; Hirsch, 1988; Dudkowski, 2016) etc. We list here just some of them, which we follow in our papers (Golubyatnikov et al., 2004; Gaidov et al., 2006; Gaidov, Golubyatnikov, 2014; Ayupova et al., 2017), see also references therein.

Our recent results were devoted to description of topological and combinatorial structures of phase portraits of the circular gene networks models, which were constructed in their simplest form in (Elowitz, Liebler, 2000). The main aim of our studies here consists of detection of periodic trajectories (cycles), determination of their locations in the phase portraits, and in finding conditions of existence, stability, uniqueness (or non-uniqueness) of these cycles. Importance of biological meaning of these questions is well-known (Kolchanov et al., 2008; Likhoshvai et al., 2020), and we shall restrict ourselves to mathematical problems which arise here naturally as well. In preparation of numerical experiments with gene networks models, one should estimate the values of their parameters. So, in our theorems we determine conditions which provide the desired qualitative and quantitative behavior of trajectories of these models.

Some of our results are connected with detection of invariant domains and invariant surfaces of phase portraits of dynamical systems which simulate gene networks functioning. Usually, these invariant domains allow to describe attractors of the dynamical systems. The invariant surfaces either are located in the boundaries of basins of attraction of these attractors, or allow to reduce the dimension of dynamical systems, and due to this reduction, the numerical experiments with the gene networks models can be simplified essentially, see (Bukharina et al., 2018; Golubyatnikov, Kirillova, 2020).

For example, following (Marnellos, Mjolsness, 1998; Furman, Bukharina, 2008), we have studied in (Akinshin et al., 2014; Ayupova, Golubyatnikov, 2017) mathematical models of interaction of two, respectively, three cells in the proneural cluster of $D$. melanogaster mechanoreceptor on early stage of its development. Phase portraits of these models contain invariant surfaces on boundaries of attraction basins of their stable equilibrium points, and for appropriate values of parameters of these models, these invariant surfaces contain unstable periodic trajectories of corresponding dynamical systems.

\section{Dynamical systems and their phase portraits}

The main aim of this paper is description of combinatorial and geometric structure of phase portrait of block-linear dynamical system

$$
\begin{aligned}
& \frac{d m_{1}}{d t}=\mathrm{L}_{1}\left(\mathrm{p}_{3}\right)-\mathrm{k}_{1} \mathrm{~m}_{1} ; \\
& \frac{d p_{i}}{d t}=\Gamma_{j}\left(m_{j}\right)-l_{j} p_{j} ; \\
& \frac{d m_{i}}{d t}=\mathrm{L}_{i}\left(\mathrm{p}_{i-1}\right)-\mathrm{k}_{j} \mathrm{~m}_{i} .
\end{aligned}
$$

This is a simple version of a regulatory switch, see (Boczko et al., 2007; Chen et al., 2018).

Here and below, $j=1,2,3 ; i=2,3$; all parameters, variables, and functions are non-negative, the monotonic step-functions $L_{j}(z), \Gamma_{j}(z)$ are defined by $L_{j}(z)=a_{j} k_{j}$ for $0 \leq z \leq 1 ; L_{j}(z)=0$ for $1<z$; they correspond to negative feedbacks;

$\Gamma_{j}(z)=0$ for $0 \leq z \leq 1 ; \Gamma_{j}(z)=b_{j} l_{j}$ for $1<z$; these functions describe positive feedbacks, see (Golubyatnikov, Minushkina, 2019). We assume in our considerations that

$$
a_{j}>1 ; b_{j}>1
$$

In smooth dimensionless symmetric case $L_{j}(z)=a\left(1+z^{\gamma}\right)^{-1}+$ $\beta_{i} \Gamma_{j}(z)=\mu z ; k_{j}=1 ; l_{j}=\mu$ for all $j$, this system appeared in (Elowitz, Leibler, 2000) as a model of one natural gene network with three different proteins TetR, $\lambda \mathrm{cl}$, Lacl, which have concentrations $p_{1}$, $p_{2}, p_{3}$, respectively. The variables $m_{1}, m_{2}, m_{3}$, denote concentrations of corresponding mRNAs. This symmetric smooth case of the system (1) was studied later in (Glyzin et al., 2016).

Since all these three proteins in the gene network are quite different, we consider here asymmetric version of the system (1): all the parameters $k_{j}, l_{j}$ are different as well as the functions $L_{j}$ and $\Gamma_{j}$.

Smooth asymmetric versions of the system (1) were examined in (Ayupova et al., 2017; Bukharina et al., 2018; Golubyatnikov, Kirillova, 2020) where conditions of existence of cycles were established.

Similar block-linear dynamical systems of different dimensions as models of gene networks functioning are considered in (Ayupova, Golubyatnikov, 2014; Golubyatnikov, Ivanov, 2018a), where the main questions concern detection of the cycles and localization of their positions in the phase portraits. The first step of these studies is determination of an invariant domain which contains the cycles, the equilibrium points, and other objects 
of interest in these portraits. It was shown in (Golubyatnikov, Minushkina, 2019) that the parallelepiped $\mathrm{Q}^{6}=\left[0, a_{1}\right] \times\left[0, b_{1}\right] \times$ $\left[0, a_{2}\right] \times\left[0, b_{2}\right] \times\left[0, a_{3}\right] \times\left[0, b_{3}\right]$ in $\mathrm{R}_{+}^{6}$ is an invariant domain for the block-linear dynamical system (1), and for its smooth analogues, see (Ayupova et al., 2017), where the smooth functions $L_{j}$ decrease monotonically (negative feedbacks), and the smooth functions $\Gamma_{j}$ are monotonically increasing (positive feedbacks). Following results of these publications, we decompose $\mathrm{Q}^{6}$ by six hyper-planes $m_{j}=1, p_{j}=1$, which contain the point $E=(1 ; 1 ; 1 ; 1 ; 1 ; 1)$ in $Q^{6}$ where right-hand sides of all equations of the system (1) have discontinuities. So, this domain $\mathrm{Q}^{6}$ is decomposed to 64 blocks; each block $B$ can be enumerated naturally by binary multi-index: $\left\{\varepsilon_{1} \varepsilon_{2} \varepsilon_{3} \varepsilon_{4} \varepsilon_{5} \varepsilon_{6}\right\}$ where

$$
\begin{aligned}
& \varepsilon_{2 j-1}=0 \text { if } 0 \leq m_{j} \leq 1 ; \varepsilon_{2 j-1}=1 \text { if } 1 \leq m_{j} \text { for all points of } B ; \\
& \varepsilon_{2 j}=0 \text { if } 0 \leq p_{j} \leq 1 ; \varepsilon_{2 j}=1 \text { if } 1 \leq p_{j} \text { for all points of } B .
\end{aligned}
$$

Analogous decompositions of the invariant domains $Q$ of quite different $n$-dimensional dynamical systems of the type (1), block-linear or smooth, with monotonic functions in righthand sides of their equations were considered in (Bukharina et al., 2018; Golubyatnikov et al., 2010; Golubyatnikov, Ivanov, 2018a). Note, that for smooth systems of this type the role of the common point $E$ of all $2^{n}$ blocks of the decomposition plays the equilibrium point of each of these systems. As it was shown in (Golubyatnikov et al., 2004, 2010; Golubyatnikov, Kirillova, 2020), this point does exist and is unique, and for any pair of incident blocks $B_{1}, B_{2}$ separated in $Q^{n}$ by $(n-1)$-dimensional hyper-face $F=B_{1} \cap B_{2}$, trajectories of all points of this intersection transit either from $B_{1}$ to $B_{2}$, i.e. $B_{1} \rightarrow B_{2}$, or in the opposite direction from $B_{2}$ to $B_{1}$, i.e., $B_{2} \rightarrow B_{1}$.

Definition (Ayupova et al., 2017). The valence $V(B)$ of a block $B$ is a number of its hyper-faces $\left\{F_{S}\right\}$ such that trajectories of the points of these hyper-faces leave the block $B$ to the incident ones.

\section{State Transition Diagrams}

We have studied in (Golubyatnikov et al., 2004, 2005) different 3D versions of the system (1):

$$
\mathrm{d} x / \mathrm{d} t=f_{1}(z)-k_{1} x ; \mathrm{d} y / \mathrm{d} t=f_{2}(x)-k_{2} y ; \mathrm{d} z / \mathrm{d} t=f_{3}(y)-k_{3} z ;
$$

where the monotonically decreasing functions $f_{j}$ are smooth or block-linear. It was shown there that the domain $\mathrm{Q}^{3}=\left[0, f_{1}(0)\right] \times\left[0, f_{2}(0)\right] \times\left[0, f_{3}(0)\right]$ in $\mathrm{R}^{3}$ is an invariant domain of the system (4), and that in its decomposition to 8 blocks enumerated by multi-indices $\left\{\varepsilon_{1} \varepsilon_{2} \varepsilon_{3}\right\}$, the blocks $\{000\}$ and $\{111\}$ are 3 -valent. The remaining 6 blocks are 1 -valent and can be arranged to a circular diagram

$$
\{001\} \rightarrow\{011\} \rightarrow\{010\} \rightarrow\{110\} \rightarrow\{100\} \rightarrow\{101\} \rightarrow\{001\} \rightarrow \ldots
$$

Here the arrows show the unique way for trajectories of the system (4) to leave these blocks and to travel in their union. For some other classes of gene networks models, similar diagrams were used in description of attractors of trajectories, and were called state transition diagrams (Glass, Pasternack, 1978), or state transition graphs (Abou-Jaoudé et al., 2016).

Note, that if the functions $f_{j}$ in the right-hand sides of the equations are not monotonic (even in the unimodal case), then trajectories of these higher-dimensional dynamical systems can exhibit chaotic behavior, see (Golubyatnikov et al., 2005; Likhoshvai et al., 2013, 2015). In the 3D case these trajectories are not so chaotic (Gaidov et al., 2010).

Now, in the case of the dynamical system (1) and its smooth analogues, all their 1-valent blocks can be arranged to the state transition diagram

$$
\begin{aligned}
& \{110011\} \rightarrow\{010011\} \rightarrow\{000011\} \rightarrow\{001011\} \\
& \uparrow \\
& \{110010\} \\
& \uparrow
\end{aligned} \quad\left\{\begin{array}{c}
0001111\} \\
\downarrow \\
\{001101\} \\
\downarrow \\
\{110000\} \leftarrow\{110100\} \leftarrow\{111100\} \leftarrow\{101100\} \leftarrow\{001100\}
\end{array}\right.
$$

Figure 1. State transition diagram composed by 1 -valent blocks of the system (1)

Let $W_{1}$ be their union. This is an invariant domain of the system (1). We have proved in (Golubyatnikov, Minushkina, 2019) that if $a_{>}>1, b_{j}>1$, see (2), then $W_{1}$ contains a cycle $\boldsymbol{C}$ which travels from block to block according to arrows of the diagram (6).

In the case of the system (4), it was shown in (Golubyatnikov, Ivanov, 2018b) that if $f_{1}(0), f_{2}(0), f_{3}(0)>1$, then the union of the 1 -valent blocks listed in (5) contain a unique cycle, and that this cycle is stable, see also (Ayupova, Golubyatnikov, 2014). Most of the steps of the proofs of these $3 D$ results on uniqueness and stability of the cycle in the union of 1-valent blocks can be extended to higher-dimensional cases, for example to the system (1). Numerical experiments (Ayupova et al., 2017; Bukharina et al., 2018; Likhoshvai et al., 2020) also confirm the hypothesis that for any-dimensional systems of this type, the union of their 1-valent blocks, such as $W_{1}$ shown on the Figure 1 , contains a unique cycle which is stable.

The invariant domain $\mathrm{Q}^{6}$ contains also 40 blocks of the valence 3 , and 12 blocks of the valence 5 , which can be arranged to a diagram (7), see the Figure 2.

In contrast with $W_{1}$, the union $W_{5}$ of all the 5 -valent blocks listed in this diagram is not an invariant domain. For example, trajectories of the points of the block $\{111001\}$ can transit to the 5-valent block $\{011001\}$, as it is shown in the diagram (7), but they can pass also to four 3-valent blocks $\{110001\}$, $\{111101\},\{111011\}$, and $\{111000\}$ which are not listed in the diagram (7).

$$
\begin{aligned}
& \{111001\} \rightarrow\{011001\} \rightarrow\{011000\} \rightarrow\{011010\} \rightarrow\{011110\} \\
& \uparrow \\
& \{101001\} \\
& \uparrow \\
& \{100001\} \leftarrow\{100101\} \leftarrow\{100111\} \leftarrow\{100110\} \leftarrow\{000110\}
\end{aligned}
$$

Figure 2. State transition diagram composed by 5 -valent blocks of the system (1) 
Theorem. If $a_{j}>2, b_{j}>3$ for all $j$, then the non-invariant domain $W_{5}$ contains an invariant piecewise linear 2-dimensional surface $M$. Trajectories of all points of this surface tend to the discontinuity point $E$ in a spiral way.

This surface $M$ is contained in the boundary of basin of attraction of the limit cycle $\mathbf{C}$.

It was shown in (Golubyatnikov, Ivanov, 2018a) that if the inequalities (2) are not satisfied, then behavior of trajectories of the system (1) and its block-linear analogues is quite trivial, there are no limit cycles, just one stable equilibrium point.

If one represents the multi-indices $\left\{\varepsilon_{1} \varepsilon_{2} \varepsilon_{3} \varepsilon_{4} \varepsilon_{5} \varepsilon_{6}\right\}$ in the diagrams (6), (7) by decimal numbers, then the arrows of these diagrams will correspond to addition of $\pm 2^{m}$ for $m=0,1,2,3,4$, or 5 . Namely, these arrows describe changes of the decimal indices of blocks as follows:

$$
\text { for (6): }-32 ;-16 ;+8 ;+4 ;-2 ;-1 ;+32 ;+16 ;-8 ;-4 ;+2 ;+1 \text {; }
$$$$
\text { for (7): }-32 ;-1 ;+2 ;+4 ;-8 ;-16 ;+32 ;+1 ;-2 ;-4 ;+8 ;+16 \text {. }
$$

In both cases, we start from the left arrows in the top rows, up to signs we have here geometrical progressions.

Similar interpretation has the diagram (5), and analogues of all these diagrams constructed in (Golubyatnikov et al., 2010; Bukharina et al., 2018; Golubyatnikov, Kirillova, 2020).

Combinatorial structure of the union $W_{3}$ of the 3-valent blocks in the invariant domain $\mathrm{Q}^{6}$ and its analogues in other dimensions is much more complicated than that of $W_{1}$ and $W_{5}$. For example, for 5-dimensional dynamical system of the type (4), considered in (Golubyatnikov et al., 2010), transitions from 3-valent blocks to 3-valent blocks is not described by just geometrical progressions as in (8). However, we conjecture that for the system (1) the domain $W_{3}$ should contain at least one invariant surface as $W_{5}$.

\section{References}

Abou-Jaoudé W., Traynard P., Monteiro P.T., Saez-Rodriguez J., Helikar T. Thieffry D., Chaouiya C. Logical modeling and dynamical analysis of cellular networks. Front. Genet. 2016;7(94):01-20. DOI 10.3389/ fgene.2016.00094

Abraham R., Robbin J. Transversal mappings and flows. New York: W.A. Benjamin Inc., 1967.

Akinshin A.A., Bukharina T.A., Furman D.P., Golubyatnikov V.P. Mathematical modeling of interaction of two cells in the proneural cluster of the wing imaging disk of $D$. melanogaster. Siberian Journal Pure Appl. Math. 2014;14(4):03-10. (in Russian)

Ayupova N.B., Golubyatnikov V.P. On the Uniqueness of a Cycle in an Asymmetric Three-Dimensional Model of a Molecular Repressilator. J. Appl. Indust. Math. 2014;8(2):153-157. DOI 10.1134/ S199047891402001X.

Ayupova N.B., Golubyatnikov V.P. A three-cells model of the initial stage of development of a proneural cluster. J. Appl. Ind. Math. 2017;11(2) 1-7. DOI 10.1134/S1990478917020028.

Ayupova N.B., Golubyatnikov V.P., Kazantsev M.V. On the existence of a cycle in an asymmetric model of molecular repressilator. Numer. Anal. Appl. 2017;10(2):101-107. DOI 10.1134/S199542391702001X.

Baer S.M., Li B., Smith H.L. Multiple limit cycles in the standard model of three species competition for three essential resources. J. Math. Biol. 2006;52:745-60. DOI 10.1007/s00285-005-0367-x.
Banks H.T., Mahaffy J.M. Stability of cyclic gene models for systems involving repression. J. Theor. Biol. 1978;74:323-334. DOI 10.1016/00225193(78)90079-6.

Boczko E., Gedeon T., Mischaikow K. Dynamics of a simple regulatory switch. J. Math. Biol. 2007;55:679-719. DOI 10.1007/s00285-0070102-x.

Bukharina T.A., Golubyatnikov V.P., Furman D.P., Kazantsev M.V., Kirillova N.E. Mathematical and Numerical Models of Two Asymmetric Gene Networks. Siberian Electron. Math. Rep. 2018;15:1271-1283. DOI 10.17377/semi.2018.15.103.

Chen H., Levo M., Barinov L., Fujioka M., Jaynes J.B., Gregor T. Dynamic interplay between enhancer-promoter topology and gene activity. Nat. Genet. 2018;50:1296-1303. DOI 10.1038/ s41588-018-0175-z.

Dudkowski D., Jafari S., Kapitaniak T., Kuznetsov N.V., Leonov G.A., Prasad A. Hidden attractors in dynamical systems. Phys. Rep. 2016;637:1-50. DOI 10.1016/j.physrep.2016.05.002.

Elowitz M.B., Leibler S. A synthetic oscillatory network of transcriptional regulators. Nature. 2000;403:335-338.

Furman D.P., Bukharina T.A. How Drosophila melanogaster Forms Its Mechanoreceptors. Curr. Genomics. 2008;8:312-323. DOI 10.2174/138920208785133271.

Gaidov Yu.A., Golubyatnikov V.P. On cycles and other geometric phenomena in phase portraits of some nonlinear dynamical systems. Geometry Applications. Springer, New York, 2014:225-233 (Springer Proc. Math. Stat.; V. 72). DOI 10.1007/ 978-3-319-04675-4_10.

Gaidov Yu.A., Golubyatnikov V.P., Kleshchev A.G., Volokitin E.P. Modeling of asymmetric gene networks functioning with different types of Regulation. Biophysics. 2006;51(suppl. 1):S61-S65. DOI 10.1134/ S000635090607013X.

Gaidov Yu.A., Golubyatnikov V.P., Likhoshvai V.A. Some Nonlinear Dynamic Systems Modeling Asymmetric Gene Networks. Part 2. Siberian Journal Pure Appl. Math. 2010;10(1):18-28. (in Russian)

Glass L., Pasternack J.S. Stable oscillations in mathematical models of biological control systems. J. Math. Biol. 1978;6:207-223.

Glyzin S.D., Kolesov A.Yu., Rozov N.Kh. Buffering in Cyclic Gene Networks. Theor. Math. Phys. 2016;187(3):935-951. DOI 10.1134/ S0040577916060106.

Golubyatnikov V.P., Golubyatnikov I.V., Likhoshvai V.A. On the Existence and stability of cycles in five-dimensional Models of Gene Networks. Numer. Anal. Appl. 2010;3(4): 329-335. Novosibirsk, Russia 10.1134/ S199542391004004X.

Golubyatnikov V.P., Ivanov V.V. Cycles in odd-dimensional models of circular gene networks. J. Appl. Ind. Math. 2018a;12(4):648-657. DOI 10.1134/S1990478918040051.

Golubyatnikov V.P., Ivanov V.V. Uniqueness and stability of a cycle in 3-dimensional block-linear circular gene network models (in Russ). Siberian J. Pure Appl. Mathem. 2018b;18(1):19-28. DOI 10.33048/ pam.2018.18.402.

Golubyatnikov V.P., Kirillova N.E. On Cycles in Models of Functioning of Circular Gene Networks. J. Math. Sci. 2020;246(6): 779-787. DOI 10.1007/s10958-020-04780-7.

Golubyatnikov V.P., Likhoshvai V.A., Gaidov Yu.A., Kleshchev A.G., Lashina E.A. Regular and chaotic dynamics in the gene network modeling. Proceedings of 8-th International conference "Human\&Computers", Japan, Aizu-Wakamatsu. 2005;7-12.

Golubyatnikov V.P., Likhoshvai V.A., Ratushny V.A. Existence of closed trajectories in 3-D gene networks. Journal Three-Dimensional Images. 2004;18(4):96-101.

Golubyatnikov V.P., Minushkina L.S. Monotonicity of the Poincaré mapping in some models of circular gene networks. J. Appl. Ind. Math. 2019;13(3):472-479. DOI 10.1134/S1990478919030086.

Hastings S., Tyson J.J., Webster D. Existence of periodic solutions for negative feedbacks cellular control systems. J. Differ. Equ. 1977;25: 39-64. 
Hirsch M.W. Systems of differential equations which are competitive or cooperative: III. Competing species. Nonlinearity. 1988;1: 51-71.

Kolchanov N.A., Goncharov S.S., Ivanisenko V.A., Likhoshvai V.A., eds. Computational Systems Biology. Novosibirsk, 2008.

Likhoshvai V.A., Fadeev S.I., Kogai V.V., Khlebodarova T.M. Alternative splicing can lead to chaos. J. Bioinform. Comput. Biol. 2015;13(1):1540003-01-25. DOI 10.1142/S021972001540003X.
Likhoshvai V.A., Golubyatnikov V.P., Khlebodarova T.M. Limit cycles in models of circular gene networks regulated by negative feedback loops. BMC Bioinform. 2020;21(Suppl 11):255.

Likhoshvai V.A., Kogai V.V., Fadeev S.I., Khlebodarova T.M. On the chaos in gene networks. J. Bioinform. Comput. Biol. 2013;11(1):134000901-1340009-25. DOI 10.1142/S021972001340009X.

Marnellos G., Mjolsness E. A Gene Network Approach to Modeling Early Neurogenesis in Drosophila. Pacific Symp. Biocomput. 1998;30-41.

Conflict of interest. The authors declare no conflict of interest.

Received August 27, 2020. Revised September 14, 2020. Accepted September 15, 2020. 